\title{
Comprehensive Characterization of the Self-Folding Cavitand Dynamics
}

\author{
Ricard López-Coll, ${ }^{[a]}$ Rubén Álvarez-Yebra, ${ }^{[a]}$ Ferran Feixas, ${ }^{*[a]}$ and Agustí Lledó ${ }^{*[a]}$
}

\begin{abstract}
The conformational equilibria and guest exchange process of a resorcin[4]arene derived self-folding cavitand receptor have been characterized in detail by molecular dynamics simulations (MD) and ${ }^{1} \mathrm{H}$ EXSY NMR experiments. A multi-timescale strategy for exploring the fluxional behaviour of this system has been constructed, exploiting conventional $\mathrm{MD}$ and accelerated MD (aMD) techniques. The use of $\mathrm{aMD}$ allows the reconstruction of the folding/unfolding process of the receptor by sampling high-energy barrier processes unattainable by conventional MD simulations. We obtained MD trajectories sampling events occurring at different time-
\end{abstract}

scales from ns to s: 1) rearrangement of the directional hydrogen bond seam stabilizing the receptor, 2) folding/ unfolding of the structure transiting partially open intermediates, and 3) guest departure from different folding stages. Most remarkably, reweighing of the biased aMD simulations provided kinetic barriers that are in very good agreement with those determined experimentally by ${ }^{1} \mathrm{H}$ NMR. These results constitute the first comprehensive characterization of the complex dynamic features of cavitand receptors. Our approach emerges as a valuable rational design tool for synthetic host-guest systems

\section{Introduction}

The term cavitand was coined by Cram and co-workers in 1982 to describe "synthetic organic compounds that contain enforced cavities large enough to accommodate simple molecules or ions". ${ }^{[1]}$ Ever since, this definition has been preferentially used for receptors obtained by covalently bridging the phenolic moieties of resorcin[4]arenes with aromatic spacers. In 1997, Rebek and co-workers introduced a significant improvement of this concept and synthesized the first self-folding cavitands, in which the binding-competent closed conformation of the receptor is stabilized by a seam of cooperative hydrogen bonding interactions between amide groups on the open rim of the cavitand (1, Figure 1). ${ }^{[2]}$ As a result, host-guest equilibria are slowed down significantly, allowing the guests to remain longer in the confined space of the receptor. This feature has been exploited to isolate elusive reactive intermediates and develop biomimetic catalysis with properly functionalized cavitands (IA, Figure 1). ${ }^{[3]}$ Inspiration from nature has been historically the main drive in the development of supramolecular catalysis, ${ }^{[4]}$ and we have focused during the last

[a] R. López-Coll, R. Álvarez-Yebra, Dr. F. Feixas, Dr. A. Lledó Institut de Química Computacional i Catàlisi (IQCC),

Departament de Química

Universitat de Girona

Maria Aurèlia Capmany 69, 17003 Girona

E-mail: ferran.feixas@udg.edu agusti.lledo@udg.edu

Supporting information for this article is available on the WWW under https://doi.org/10.1002/chem.202100563

of $\odot 2021$ The Authors. Chemistry - A European Journal published by Wiley$\mathrm{VCH} \mathrm{GmbH}$. This is an open access article under the terms of the Creative Commons Attribution Non-Commercial NoDerivs License, which permits use and distribution in any medium, provided the original work is properly cited, the use is non-commercial and no modifications or adaptations are made.
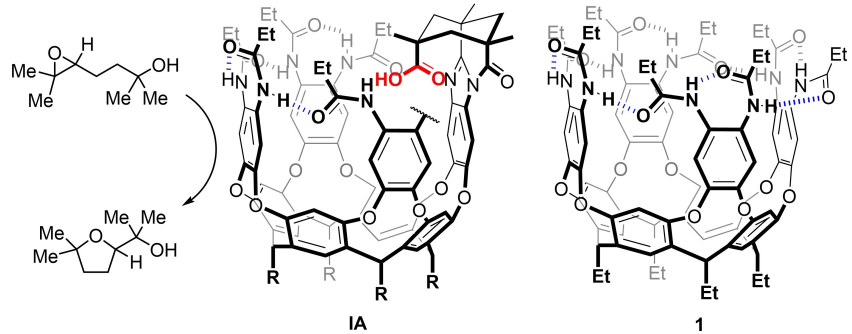

Self-folding cavitands

- Demonstrated potential in enzyme-like catalysis

- Complex fluxional behavior

- Poor understanding of exchange dynamics

Figure 1. The self-folding introverted acid cavitand (IA) displays enzyme like catalysis of epoxy-alcohol cyclization reactions. The self-folding parent cavitand structure $\mathbf{1}$ is used in this work to study the guest exchange dynamics of these systems, in combination with 1-adamantanecarbonitrile (2) as a guest.

years on developing new functionalized receptors for this purpose. ${ }^{[5]}$ With notable exceptions, the de novo development of supramolecular catalysis with cavitands and analogous receptors is plagued with a number of impediments, such as product inhibition. ${ }^{[6]}$ The design of appropriate guests or substrates is also far from trivial, and relies primarily on qualitative and empirical considerations such as the $55 \%$ rule, of uncertain validity for open-end containers. ${ }^{[7]}$ In this scenario, the use of computational tools appears as a necessity to unveil the bases of the molecular recognition events that govern these systems. Only then can these first principles be applied to the rational design of new host-guest systems suitable for catalytic applications.

A number of theoretical studies on resorcin[4]arene-derived receptors have been reported, mostly based on the character- 
ization of stationary points employing ab-initio methods. ${ }^{[8]}$ Arguably, this approach fails to grasp the complex dynamic nature that is intrinsic to most host-guest systems. Cavitands, for instance, are capable of displaying a range of different conformations in solution, and this fluxional behaviour is key for the molecular recognition process. However, this intrinsic flexibility remains unexplored on the molecular detail. Given the established parallels of self-folding cavitands with enzymatic receptors, we propose to use Molecular Dynamics (MD) simulations - the method of choice for modelling protein dynamics - to reconstruct in detail the binding and exchange dynamics of the said host-guest complexes, laying the foundations for a universal and computationally guided approach to the design of synthetic host guest systems for different purposes, including supramolecular catalysis. In this particular area, the use of MD simulations has recently emerged as an invaluable modelling tool. ${ }^{[9]}$

Self-folding cavitand receptors display conformational and guest exchange processes with relatively high barriers $(\Delta G)$, in the range of $16-19 \mathrm{kcal} \mathrm{mol}^{-1},{ }^{[10]}$ corresponding to timescales of milliseconds to seconds. ${ }^{[1]]}$ These timescales are still not accessible for conventional MD simulations: low-energy states relevant to these processes are separated by high-energy barriers in the free-energy landscape, which are rarely crossed over the course of a single unbiased MD simulation. ${ }^{[12]}$ Recently, the exchange dynamics of related non-hydrogen bonded cavitands with lower conformational interconversion barriers have been studied. ${ }^{[13]}$

A proper depiction of a complex system involving motions with disparate energy barriers and time scales necessitates a combination of MD-based techniques tailored to these individual events, including enhanced sampling techniques for exploring transitions involving high barrier processes. In this regard, MD simulations can be seen as a "computational microscope" with different "objectives" for each of the required time scales. Enhanced sampling techniques represent an attractive strategy to sample slow processes that cannot be explored by conventional MD simulations. ${ }^{[14]}$ The basis of speeding up conformational sampling is the introduction of an artificial bias into the model upon which the MD simulations are based. Enhanced sampling methods can be divided into two groups: those based on biasing a set of collective variables (CVs), and unconstrained methods. Using a refined set of CVs, Pavan and co-workers recovered from metadynamics simulations the thermodynamic and kinetics of guest binding and release in a coordination cage, obtaining results in agreement with experimental values. ${ }^{[15]}$ However, the identification of appropriate CVs can become complex when relevant states in the studied process remain hidden. Among the unconstrained methods, accelerated molecular dynamics (aMD) is particularly appealing because it does not rely on the a priori definition of a set of CVs and, therefore, provides unconstrained enhanced sampling to freely explore the molecular conformational space. ${ }^{[16]}$ aMD enhances conformational sampling by adding a non-negative boost potential to the system when the system potential is lower than a reference energy. Recently, aMD simulations have been successfully employed to explore the folding pathways of small proteins, and to reconstruct substrate binding in enzymatic and supramolecular receptors. ${ }^{[1]]}$

Self-folding cavitands such as 1 display a distinct conformational behaviour (Scheme 1). Two extreme conformations exist: the vase form or closed conformer (V), which is competent for binding smaller complementary molecules, and the kite open conformer (K). ${ }^{[18]}$ The latter features an extended flat aromatic surface and is ripe for self-aggregation through $\pi$-stacking interactions. The resulting velcrand dimer prevails over higher order aggregates and is devoid of further molecular recognition properties. Guest exchange is in slow regime compared to the NMR time scale, producing two sets of signals for bound and unbound guest resonances. The bound guest's resonances experience large anisotropic shielding from the cumulative effect of 8 aromatic panels, resulting in large upfield shifts. As a result, aliphatic protons on the guest appear in the far upfield region of the ${ }^{1} \mathrm{H}$ NMR spectrum, which is devoid of interference
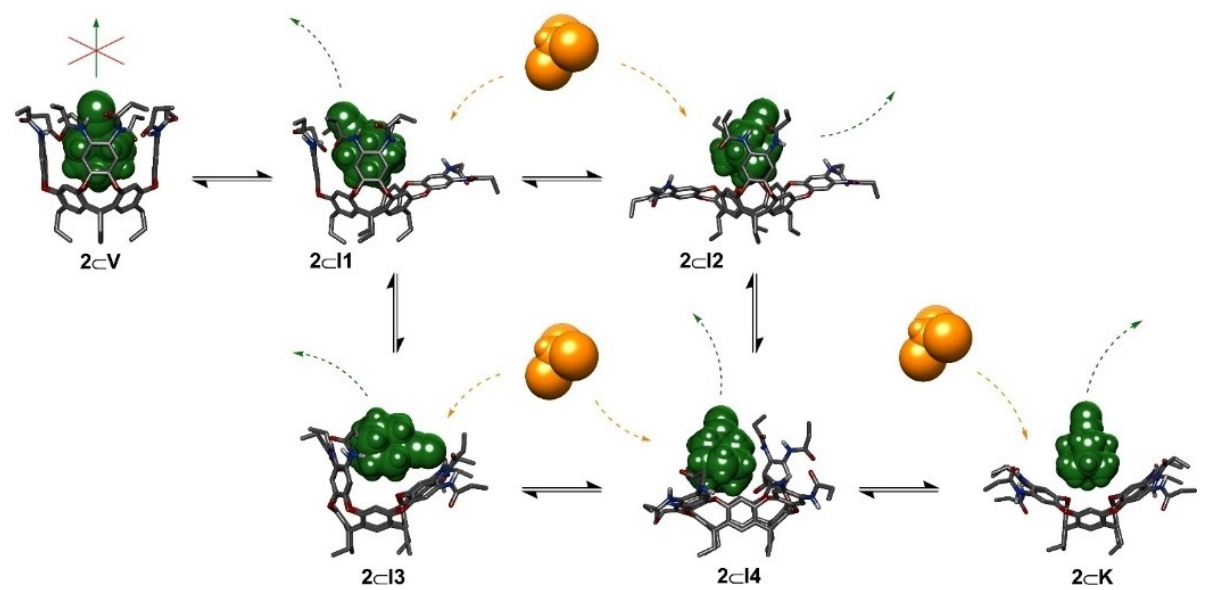

Scheme 1. All possible conformational equilibria involved in cavitand folding/unfolding and guest exchange. $\mathbf{V}$ (vase), and $\mathbf{K}$ (kite) refer to the extreme open and closed conformers of 1. 11-14 refer to the intermediate partially opened conformers. The arrows depict potential guest exchange by solvent molecules. Green: 1-adamantanecarbonitrile (2); orange: chloroform. The structures have been optimized at the semiempirical PM6 level of theory, including solvent effect corrections of chloroform solution. 
by the host's resonances. This feature facilitates analysis of the exchange kinetics and allows extraction of exchange rates and barriers through EXSY experiments. ${ }^{[10]}$ Importantly, no stable intermediates of the exchange process have been observed in solution by ${ }^{1} \mathrm{H}$ NMR.

Herein, we apply for the first time accelerated molecular dynamics (aMD), in combination with conventional MD, to describe in full the conformational and guest departure dynamics of the prototypical self-folding cavitand 1, using 1adamantanecarbonitrile (2) as guest in chloroform solution. We have also studied this system experimentally and extracted actual exchange rates and barriers from ${ }^{1} \mathrm{H}$ EXSY experiments, which compare favourably to values computed with our method.

\section{Results and Discussion}

At the onset of our study, we computed the structures of the $2 \subset 1$ host-guest complex in all its possible folding states. In addition to the extreme vase (V) and kite (K) forms, intermediate structures in which one, two, or three walls of the cavitand have flipped out (11-14) can be located as stable local minima by means of semiempirical calculations (Scheme 1). These structures are in good agreement with those previously found for non-hydrogen bonded congeners of $1,{ }^{[13]}$ and were used as starting geometries for the MD simulations developed thereafter.

In the following sections, we discuss the motions experienced by the system of study in different timescales, namely (from fastest to slowest respectively): a) guest departure from partially open conformers, b) concerted rotation of the amide groups on the rim of the cavitand (amide flip), and c) interconversion among different conformers by folding/unfolding of the cavitand panels. While the first event can be sampled by conventional MD simulations, the other two have been carried out with aMD methods.

\section{Guest exchange}

The following model has been proposed for guest exchange. Direct removal of the guest from the vase form $(\mathbf{V}$, Scheme 1$)$ is not viable: it would generate a void behind. Direct substitution by another guest molecule without unfolding is also unlikely, because of the crowded environment around the bound guest. An alternative scenario involves unfolding of the cavitand's walls and exposure of the guest to the surrounding medium, triggering the exchange by another guest or solvent molecule. In this process, the initial unfolding is a slow, rate-determining step that is followed by a rapid, barrier-less guest replacement. The question remains, however, how many walls must flip before the guest can freely diffuse out? To ascertain this we performed conventional MD simulations of the molecular complex of $\mathbf{2}$ with $\mathbf{1}$ in its different states, including partially open intermediates (Scheme 1): vase (V), one wall open (I1), two opposing walls open (12), two adjacent walls open (I3), three walls open (14) and kite (K). We employed as starting geometries the PM6-optimized molecular complexes of $\mathbf{2}$ with the different conformers of 1 (Scheme 1) ${ }^{\left[{ }^{[19]}\right.}$ In total, five replicas of $1 \mu \mathrm{s}$ of MD simulations for each system were carried out, gathering a total simulation time of $30 \mu \mathrm{s}$. The resulting trajectories reveal that the conformational transitions involved in the unfolding process (wall flips) are not accessible in this microsecond time scale. The wall flip transitions involve the rotation of the two dihedral angles defined by each of the junctions of the upper aromatic panels with the resorcin[4] arene core (Figure S5). These results confirm that the guest cannot escape from the vase conformer (Figure 2). In the closed conformer, the guest freely rotates inside the cavitand with the cyano group of the guest establishing transient hydrogen bonds with the amide groups. To illustrate and compare the guest exchange step along the simulations of different conformers, we monitored the distance between the centre of mass of guest 2 and the centre of the 4 upper carbons on the resorcinarene core of cavitand 1, d(1-2) (Figure 2). As with the vase conformer $(\mathbf{V})$, the guest also remains in the cavity for the duration of the simulation with conformer I1, which is in good agreement with the fact that cavitands lacking a $4^{\text {th }}$ wall provide kinetically stable host-guest complexes in the microsecond time-scale. ${ }^{[10 a, 20]}$ In this particular case, the guest is retained inside the host by means of hydrophobic interactions with the aromatic panels, and by a relatively stable hydrogen bond between the cyano group and an amide $\mathrm{NH}$ that remains available because of the removed wall (Figure S3). Interestingly, conformers with only two walls open $(\mathbf{1 2}, 13)$ allow guest replacement by solvent molecules in these simulations, with a clear transition of 2 from bound to unbound occurring in the range of $60-140$ and 5-20 ns respectively (Figures $2 c$ and S3). The conformer with two opposing walls open (12) has better shape complementarity for $\mathbf{2}$ and establishes stronger interactions with the guest than I3. In one of the replicas calculated for $\mathbf{1 2}$, the guest remains bound between the closed aromatic panels for the duration of entire simulation $(1 \mu \mathrm{s})$. For comparison, the trajectories for conformers 14 and $\mathrm{K}$ (kite) display guest $\mathbf{2}$ freely diffusing away from $\mathbf{1}$ from the very first frames of the simulation (during the equilibration phase actually), indicating a barrier-less scenario. Our results point out that the conformational equilibrium between $\mathbf{I}$ and $\mathbf{I} \mathbf{2}$ can play a key role on guest exchange and molecular recognition.

\section{Amide flip: cycloenantiomer interconversion}

Having established that cavitand unfolding requires access to high energy states not populated in the course of conventional MD simulations, we resorted to enhanced sampling techniques in order to explore the wall flip transitions that regulate folding and, ultimately, the overall guest exchange process. We applied aMD to simulate host 1 in the vase conformer using chloroform as a solvent and guest. At the onset, an average dihedral boost potential of $117 \mathrm{kcal} \mathrm{mol}^{-1}$ was applied through $14 \mu \mathrm{s}$ of aMD simulation time. This simulation protocol allows for an extensive conformational sampling of the folded state of the cavitand. 
a. Guest exchange Vase and $\mathbf{1 1}$ conformers

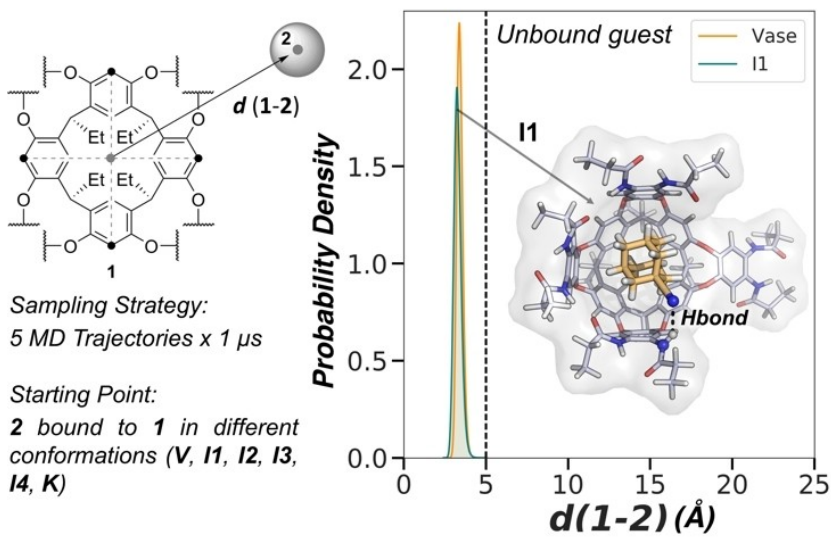

b. Guest exchange 12, 13, 14, and Kite conformers

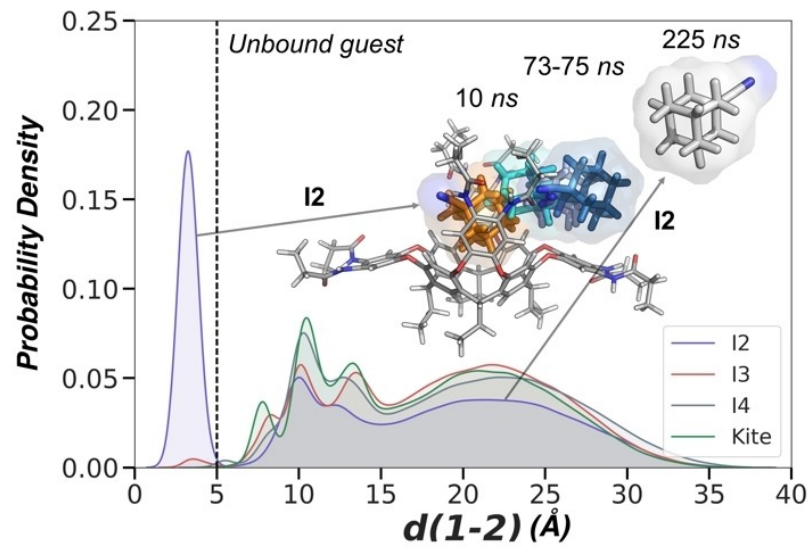

c. MD trajectories showing guest unbinding from $\mathbf{I 2}$ conformer

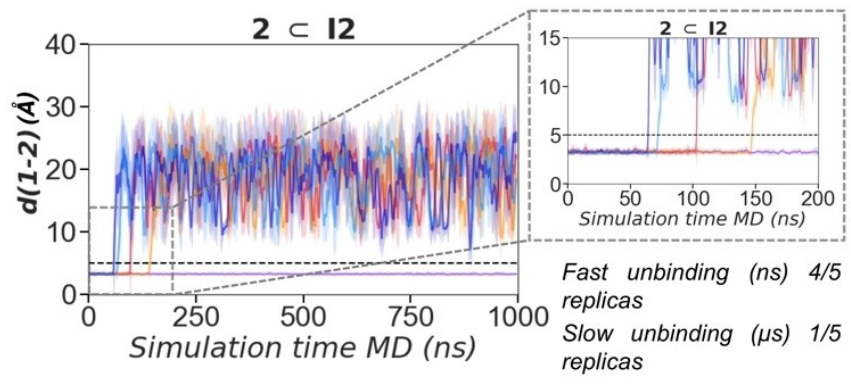

Figure 2. a) Schematic illustration of the distance $d(1-2)(\AA \AA)$ used to monitor guest departure. Probability density distribution of $d(1-2)(\AA ̊)$ for the vase (orange) and I1 (teal) conformers. Distances above $5 \AA$ indicate guest 2 unbinding. Representative snapshot of I1 MD trajectory with the hydrogen bond between the cyano group of $\mathbf{2}$ and $\mathrm{NH}$ amide of $\mathbf{1}$ highlighted in black. b) Probability density distribution of $\mathrm{d}(\mathbf{1 - 2})$ for the $\mathbf{1 2}$ (purple), 13 (red), 14 (gray), and kite (green) conformers. Representative snapshots of an MD trajectory of guest exchange for $\mathbf{1 2}$ conformer. Frames are displayed at 10 (orange), 73 (cyan), 74 (light purple), 75 (dark blue), and 225 ns (white). c) Plot of the distance $\mathrm{d}(\mathbf{1 - 2})(\AA)$ along the MD trajectories of $\mathbf{I 2}$ conformer. The distance is monitored for five $1 \mu$ s replicas (shown in different colours). A $5 \AA$ threshold is shown to highlight guest departure.

During the course of this simulation no wall flip transitions were observed. Two chloroform molecules are accommodated at the wider open end of the container, which are continuously replaced by molecules of the solvent cage throughout the simulation. A third molecule of chloroform that is bound at the tapered end of $\mathbf{V}$ in the beginning of the simulation remains inside the cavity during the whole aMD trajectory. Therefore, we can consider that guest exchange is not observed under these conditions. However, an interesting concerted motion of the amide groups forming the hydrogen bond seam is captured (Figure 3). The hydrogen bond seam defined by the amide groups is directional, imparting $C_{4}$ symmetry to the cavitand structure. Rotation about the aryl-nitrogen bond of all the amide groups provides two cycloenantiomeric forms, ( $\Delta)-\mathbf{V}$ and $(\Lambda)-\mathbf{V}$, of the cavitand (Figure 3a). The carbonyl groups are arranged clockwise ( $C$ to $O$ ) in the $\Delta$ enantiomer and anticlockwise in the $\Lambda$ enantiomer. These cycloenantiomers can interconvert by unfolding followed by amide rotation about the aryl-N bond and refolding. However, the aMD simulations unveil a concerted motion of the 8 amide groups that interconverts the cycloenantiomers without any wall flipping out. To describe the nature of this interconversion in more detail, we reconstructed the conformational landscape by applying principal component analysis (PCA, see computational methods section) to our aMD simulation (Figure $3 \mathrm{~b}$ ). Interestingly, the reconstructed conformational landscape reveals only two symmetric minima. The inspection of the structures belonging to each free energy basin shows that one minimum corresponds to ( $\Delta)-\mathbf{V}$ and the other to its cycloenantiomer $(\Lambda)-\mathbf{V}$. The absence of additional metastable minima showcases the fast concerted interconversion of the whole hydrogen bond network formed by the amide groups. The structures located in the transition region between $(\Delta)-\mathbf{V}$ and $(\Lambda)-\mathbf{V}$ remain in vase-like conformations, indicating that the interconversion takes place without partial unfolding. A detailed analysis along the aMD trajectory of the $\mathrm{NH}$...O distances involved in hydrogen bonding $\left(\mathrm{d}_{1}\right.$ and $d_{2}$, Figure 3a) shows that three transitions are observed in the timespan of the $14 \mu \mathrm{s}$ aMD simulation (Figure 3c). Distances $d_{1}$ and $d_{2}$ alternate between ca. 2 and 5-7 $\AA$ upon amide rotation. The cycloreversion process must have a significantly lower energy barrier than any unfolding motion, not observed throughout the simulations under these conditions. It is known that aMD simulations of a few microseconds of are able to capture millisecond-timescale events in proteins. ${ }^{[14 b]}$ The reweighting of the aMD simulations reveals a cycloenantiomer interconversion barrier of nearly $14.5 \mathrm{kcal} \mathrm{mol}^{-1}$ (Figure S4). All these observations are in good agreement with the fact that host-guest complexes of 1 in hydrogen bonding solvents such as acetone- $d_{6}-$, retain slow guest exchange kinetics (in the NMR time scale), while displaying fast exchange for the amide $\mathrm{NH}$ resonances. ${ }^{[10 a]}$ On the contrary, our findings disagree with the seminal work of Rebek, who reported coincident barriers for both the amide flip motion and the guest exchange process, in this case in a non-competitive apolar solvent (mesitylene- $\left.d_{12}\right) .^{[2 \mathrm{~b}]}$ Given the fact that those exchange barriers were extracted in part from coalescence experiments, we decided to re-evaluate them experimentally employing only the more accurate and well-established EXSY experiment. ${ }^{[10,21]}$ We assessed the $1^{\text {st }}$ order rate constants for the amide flip of 1 in $\mathrm{CDCl}_{3}$, and for the guest dissociation processes of $2 \subset 1$ in the 
a. Illustration of cycloenantiomer interconversion

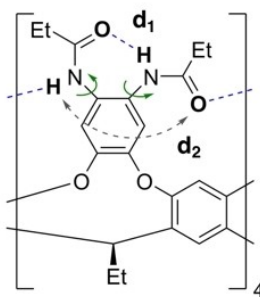

$(\Delta)-\mathbf{V}$

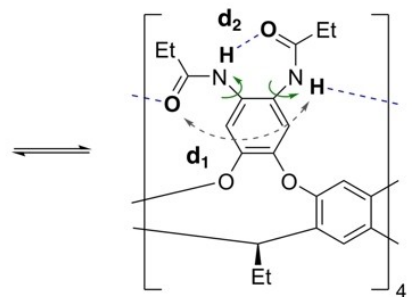

(1)-V b. Vase Conformational Landscape from aMD simulations

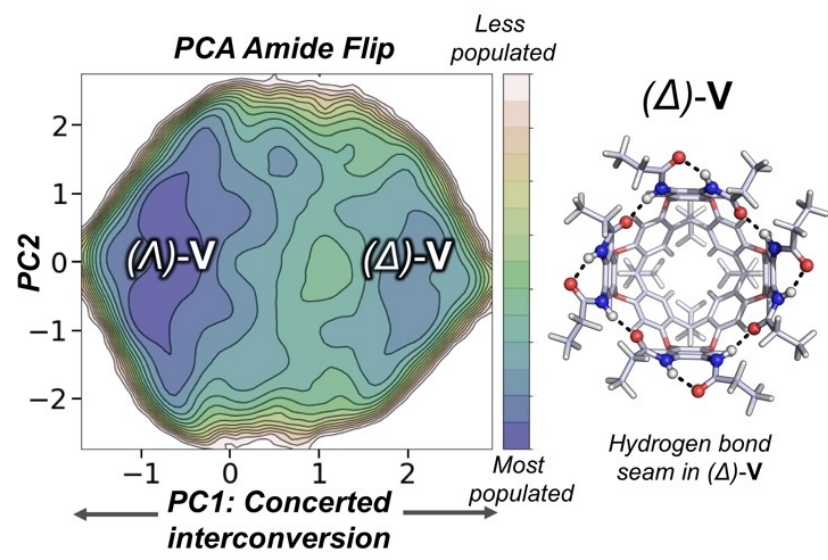

c. Concerted interconversion along the aMD simulations

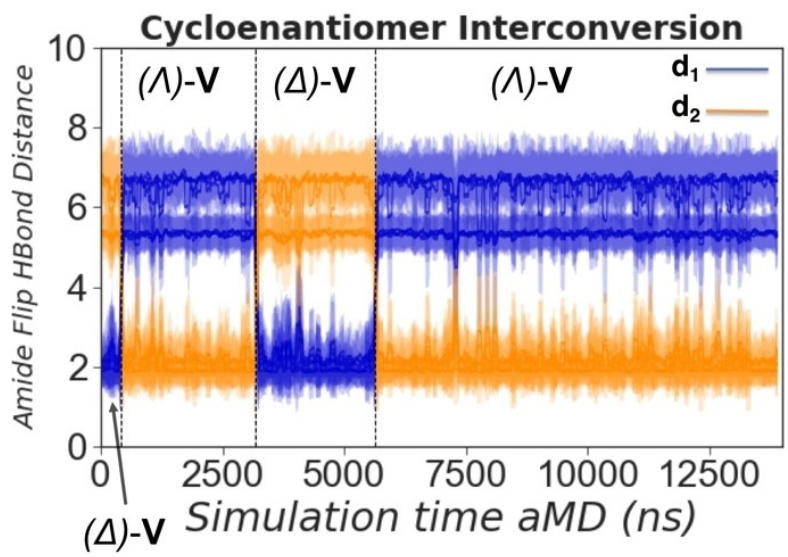

Figure 3. a) Schematic illustration of cycloenantiomer interconversion highlighting the relevant aryl- $\mathrm{N}$ bond rotation and $\mathrm{NH}$-..O distances. b) Reconstructed Conformational Landscape obtained from the PCA of accumulated simulation time of $14 \mu$ s of aMD simulations. PC1 describes the concerted motion of the eight hydrogen bonds forming the seam of the vase conformer. c) Distance plot of the two possible hydrogen bonding arrangements of each aromatic panel represented by $\mathrm{NH} \cdots \mathrm{O}$ distances $\mathrm{d}_{1}$ and $d_{2}(\AA)$, along the aMD trajectory. The plot contains the eight hydrogen bond distances in $\mathrm{V}$.

same solvent. For accuracy, we carried out ${ }^{1} \mathrm{H}$ EXSY experiments at different temperatures, and extracted the activation parameters by Eyring analysis of the rate constants at different temperatures (Figure 4). Extrapolation to $300 \mathrm{~K}$ gave exchange barriers $\Delta G$ of 13.5 and $20.0 \mathrm{kcal} \mathrm{mol}^{-1}$ for the amide flip and guest exchange respectively. These results point to significant rate differences between the two processes, even in a non-
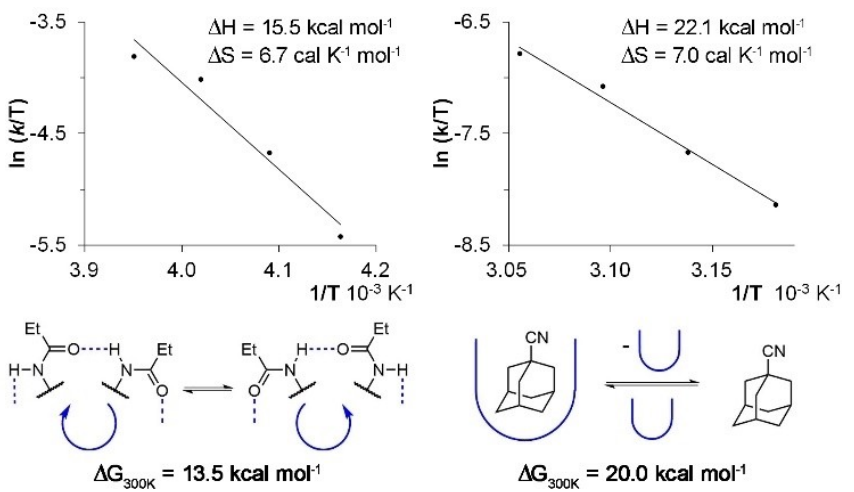

Figure 4. Eyring plots, activation parameters and free energy barriers at $300 \mathrm{~K}$ for the amide flip (left) and guest exchange processes.

hydrogen-bonding solvent, in very good agreement with our aMD simulations. Remarkably, the calculated barrier for the amide flip motion is within $1 \mathrm{kcal} \mathrm{mol}^{-1}$ difference of the experimentally determined value.

\section{Cavitand folding and unfolding}

Wall flip motions leading to unfolding or folding of the cavitand structure involve synchronous rotation of multiple dihedral angles restricted by the bridging aromatic panels. The guest exchange barrier of $20 \mathrm{kcal} \mathrm{mol}^{-1}$ determined experimentally is indicative of the energetic cost of the folding and unfolding process. In order to populate the high energy states involved in folding and cross the high energy barriers required for wall flipping, we first performed aMD simulations using significantly larger dihedral boost potentials than those used for the study of the amide flip motion. We submitted a series of $1 \mu \mathrm{s}$ aMD simulations starting from the six conformers (V, 11, 12, 13, 14, and $\mathbf{K})$ in a chloroform solvent cage and a chloroform molecule as the guest, and found out that dihedral boost potentials in excess of $1000 \mathrm{kcalmol}^{-1}$ were necessary to sample the correlated torsions required for folding. All aMD simulations converged to the vase conformer irrespective of the starting geometry, suggesting that the most populated state in solution is the vase. This observation is in good agreement with the established notion that the folded conformer is significantly more stable than the others in solution. While these forceful aMD simulations provided a qualitative picture of the selffolding process, the high and physically unrealistic average boost potentials applied prevented any kind of quantitative assessment of the folding process. A qualitative inspection of the resulting trajectories at the molecular level revealed abundant aberrant motions (e.g., exacerbated out of plane torsions in aromatic rings), corroborating that further analysis of metastable intermediates is not possible with this coarse model. To overcome this shortcoming, we set out to tailor the dualboost aMD technique to our problem. Unfortunately, previous applications to synthetic host-guest systems using specific sets of acceleration parameters are virtually non-existent. The main 
difficulty is that aMD was originally developed for proteins, and the range of $E$ (reference value) and $\alpha$ (acceleration factor) parameters that are relevant to acceleration is neither benchmarked nor fitted for non-proteinogenic systems. ${ }^{[16]}$ Additionally, the connection of $E$ and $\alpha$ to the outcoming boost and how the forces are rescaled (force weight, FW) is undetermined.

To address this, we screened a large number of different E/ $\alpha$ combinations starting from different conformers of 1 . After much experimentation, we found a set of parameters that enabled the gentle sampling of the full conformational transition - from $\mathbf{K}$ to $\mathbf{V}$ - with a moderate average dihedral boost potential of $130 \mathrm{kcalmol}^{-1}$ and total boost potential of $160 \mathrm{kcalmol}^{-1}$. Five $2.5 \mu \mathrm{s}$ simulation replicas of dual-boost aMD where run under this optimized conditions, providing reproducible results and identical outcome. Following the trend indicated by our preliminary aMD analysis, all simulations spontaneously converge to the vase conformer (V) with a chloroform molecule occupying the centre of the cavity. Under these refined conditions, smooth conformational changes leading to the closure of the cavitand are revealed, transiting through a series of interconnected metastable states.

In order to visualize and understand the folding pathway, we reconstructed the conformational landscape of the kite (K) to vase (V) folding process by applying PCA to our aMD simulations using the dihedral angles of the bridging aromatic panels as features (Figure 5, see computational methods section). The first two principal components clearly differentiate between unfolded and folded states. PC2 is directly related to the self-folding event, connecting the $\mathbf{K}, \mathbf{1} \mathbf{2}, \mathbf{I} \mathbf{1}$ and $\mathbf{V}$ conformers characterized by the progressive wall flipping. PC1 represents the concerted rotation of the two dihedrals comprising a single wall without involving the flip of the wall. Moreover, we are able to identify in the resulting conformational landscape several key intermediate states that connect the kite and vase conformations. Some of them are similar to the minima previously obtained by means of semiempirical calculations, but other relevant states are also populated, including additional I1and I2-like conformers (Figures 5a and S6). As a general trend, we observed that the folding pathway involves an initial transition from kite-like states to $\mathbf{1 2}$, where two opposite walls move up, trapping chloroform molecules in between. Then, the two remaining open walls fold in a stepwise fashion, populating first I1 and finally V. Interestingly, the PCA analysis reveals a conformational equilibrium along PC1 between $\mathbf{1 1}$ and I1' conformers. These two metastable states differ on the dihedral angle that remains unflipped in a particular wall. The concerted interconversion between these two forms precedes the formation of $\mathbf{V}$. Once the $\mathbf{V}$ form is attained, multiple amide flips are observed without unfolding of the cavitand structure. The reweighting of the aMD simulations along $\mathrm{PC2}$ indicates that the barrier that separates 12 and $\mathbf{V}$ is roughly $20 \mathrm{kcal} \mathrm{mol}^{-1}$ (Figure S4), $5.5 \mathrm{kcal} \mathrm{mol}^{-1}$ higher than the one obtained for the cycloenantiomer interconversion. These values are in very good agreement with the EXSY experiments, which indicate a similar difference between the guest exchange and the amide flip barriers $(\Delta \Delta G)$. Our results corroborate that unfolding of at least

a. Representative Cavitand Folding Landscape and Molecular Representation of Metastable States
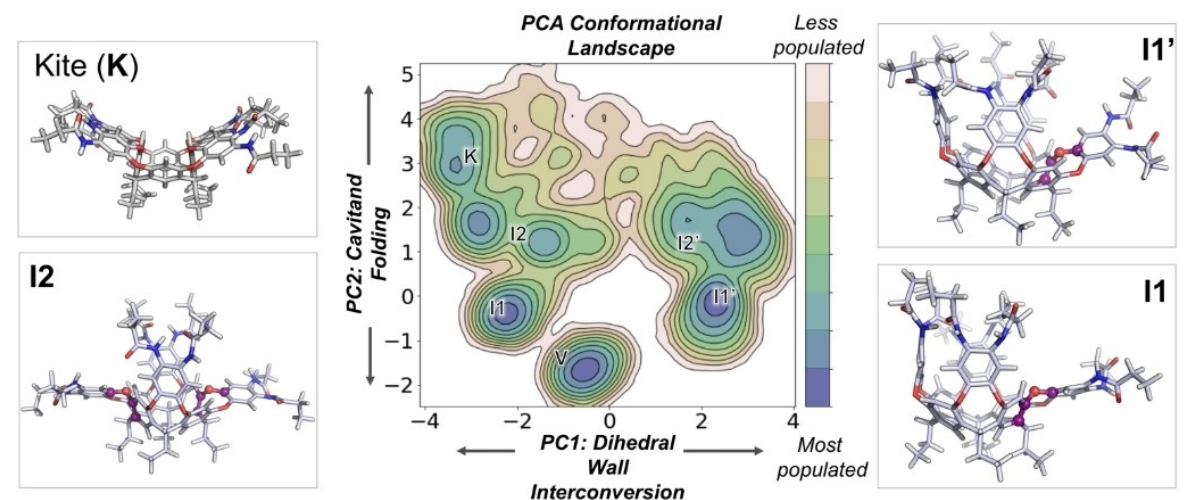

b. Principal Components along the aMD Trajectories
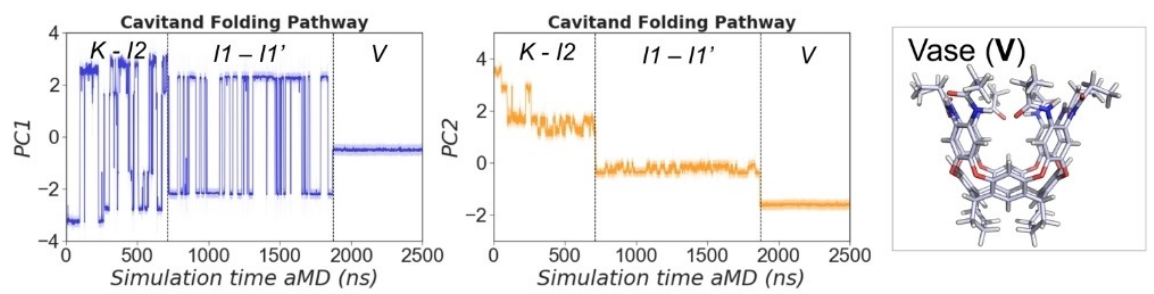

Figure 5. a) Illustration of cavitand folding pathways. Reconstructed conformational landscape obtained from principal component analysis of dihedral angles of bridging aromatic groups of an accumulated simulation time of $2.5 \mu \mathrm{s}$ of aMD simulations starting from K. PC1 represents the concerted rotation of the two dihedrals comprising a single wall without involving the flip of the wall. PC2 is directly related to the self-folding event, connecting the K, I2, I1 and V conformers characterized by the progressive wall flipping. The most representative structures are highlighted. b) Plot of two principal components along the aMD trajectory. 
two panels (as in 12) is required to complete the guest exchange process.

\section{Conclusion}

We have reproduced the guest exchange and conformational equilibria of stabilized self-folding cavitand 1 with MD simulations. We applied for the first time aMD techniques and long MD trajectories to self-folding cavitands, allowing sampling of high-energy states and conformational barriers. The aMD simulations reveal a series of hidden states not located as stationary points. Overall, our multi-timescale simulations provide an accurate description of the folding, unfolding and guest exchange processes of amide stabilized self-folding cavitands in very good agreement with experimentally determined kinetic barriers. While aMD provides excellent conformational sampling, it usually suffers in estimating the free energy barriers between different states of a large system (open/closed or bound/unbound). Our work demonstrates for the first time the use of aMD for obtaining a quantitative model of a dynamically complex host-guest system.

This method should find applicability in other systems of similar complexity, and thus become a valuable tool for the rational design of functional synthetic hosts. In the specific area of supramolecular catalysis, an accurate understanding of the guest exchange processes and their timescales could be paramount to rationalize the emergence of turnover in de novo designs. In addition to the thermochemical accuracy demonstrated in our example, our unconstrained approach has the potential to unveil hidden dynamic and exchange processes not immediately apparent when designing a functional hostguest system from scratch.

\section{Experimental Section}

Cavitand 1 was prepared following established procedures. ${ }^{[22]} 1$ Adamantanecarbonitrile (2, 97\%) was purchased from SigmaAldrich, and used as received. Details for the EXSY experiments and the calculation of the exchange rates are provided in the Supporting Information.

\section{Computational methods}

Geometries of the starting stationary points for MD simulations (conformers V, 11-14, K) with 2 as a guest were optimized without symmetry constraints with the Gaussian 09 program $^{[23]}$ at the semiempirical PM6 level, including solvent effects corrections of chloroform solution computed with the polarizable continuum solvation model (PCM). ${ }^{[24]}$

Host-Guest parameterization. The MD simulation parameters for 1 and $\mathbf{2}$ were generated within the ANTECHAMBER module of AMBER 16 using the upgraded version of the general AMBER force field (GAFF2), ${ }^{[25]}$ with partial charges calculated according to AM1-BCC scheme ${ }^{[26]}$ using ANTECHAMBER.

Conventional Molecular Dynamics Simulations. Each system was immersed in a pre-equilibrated cubic box of chloroform molecules with an internal offset distance of $12 \AA$, using the AMBER 16 LEaP module. All calculations were done using the GAFF2 force field. A two-stage geometry optimization approach was performed. First, a short minimization of the chloroform molecules positions, with positional restraints on solute by a harmonic potential with a force constant of $500 \mathrm{kcal} \mathrm{mol}^{-1} \AA^{-2}$ was done. The second stage was an unrestrained minimization of all the atoms in the simulation cell. Then, the systems were heated using six 50 ps steps, incrementing the temperature $50 \mathrm{~K}$ each step $(0-300 \mathrm{~K})$ under constant-volume, periodic-boundary conditions and the particle-mesh Ewald approach $^{[27]}$ to introduce long-range electrostatic effects. For these steps, a 10 A cut-off was applied to Lennard-Jones and electrostatic interactions. Bonds involving hydrogen were constrained with the SHAKE algorithm. Harmonic restraints of $10 \mathrm{kcal} \mathrm{mol}^{-1}$ were applied to the solute, and the Langevin equilibration scheme was used to control and equalize the temperature. The time step was kept at $2 \mathrm{fs}$ during the heating stages, allowing potential inhomogeneities to self-adjust. Each system was then equilibrated for 2 ns with a 2 fs timestep at a constant pressure of $1 \mathrm{~atm}$ (NPT ensemble). Then MD simulations and aMD simulations were performed under the NVT ensemble and periodic-boundary conditions. In total, five replicas of $1 \mu \mathrm{s}$ each of MD starting from 6 different conformers $(\mathbf{V}, \mathbf{1 1}, \mathbf{1 2}, \mathbf{1 3}$, 14 , and $\mathrm{K}$ ) have been performed with 1 -adamantanecarbonitrile bound (gathering a total of $30 \mu$ s of MD simulation).

Accelerated Molecular Dynamics Simulations. Accelerated Molecular Dynamics simulations $(\mathrm{aMD})^{[16]}$ were used to explore the cavitand dynamics and folding process using 1 and chloroform molecules as guest. The starting geometries were generated from the final step of a previous $100 \mathrm{~ns}$ conventional MD simulation. A dihedral boost potential of ca. $117 \mathrm{kcalmol}^{-1}$ was applied to simulate the interconversion of cycloenantiomers, while a finely tuned dual-boost of ca. $290 \mathrm{kcal} \mathrm{mol}^{-1}$ was used to simulate the folding process. Details for the optimization of the acceleration parameters are given in the Supporting Information. The exponential reweighting of the aMD simulations was performed using the PyReweighting toolkit. ${ }^{[28]}$ One replica of $14 \mu \mathrm{s}$ was used to characterize the cycloenantiomer interconversion. Five replicas of 1 $\mu$ s each starting from 6 different conformers ( $\mathbf{V}, \mathbf{1 1}, \mathbf{1 2}, \mathbf{1 3}, \mathbf{1 4}$, and $\mathrm{K}$ ) were performed to sample the folding pathway using high boost potential. Finally, five replicas of $2.5 \mu \mathrm{s}$ of dual-boost aMD each starting from $\mathrm{K}$ were carried out with finely tuned parameters to explore the complete folding pathway. The conformational landscape for the cycloenantiomer interconversion has been reconstructed from the accumulation of $14 \mu \mathrm{s}$ of aMD simulations. The high dimensional aMD data was reduced using principal component analysis (PCA) considering all the atoms of the cavitand. The conformational landscape for the folding and unfolding of the cavitand have been reconstructed from the accumulation $2.5 \mu$ s of dual-boost aMD simulations starting from the kite conformer. The high dimensional $\mathrm{aMD}$ data was reduced using the principal component analysis (PCA) considering all the dihedral angles of the bridging aromatic panels (Figure S5).

\section{Acknowledgements}

We are grateful for financial support from the Spanish government ("Ramón y Cajal" contract RYC2012-11112 and grant CTQ2017-83587-P to A.L.; grant RTI2018-101032-J-I00 to F.F), the European Community (MSCA-IF-2014-EF-661160-MetAccembly grant to F.F), and the Generalitat de Catalunya (projects 2017-SGR-39 and 2017-SGR-1707). We thank the Spanish Supercomputing Network (RES) for access to supercomputing resources (projects QCM-2018-3-0036, QCM-2019-1-0022, and QSB-2020-1-0008). 


\section{Conflict of Interest}

The authors declare no conflict of interest.

Keywords: Cavitands · Conformational analysis - EXSY · Hostguest systems $\cdot$ Molecular dynamics

[1] J. R. Moran, S. Karbach, D. J. Cram, J. Am. Chem. Soc. 1982, 104, 58265828.

[2] a) D. M. Rudkevich, G. Hilmersson, J. Rebek, J. Am. Chem. Soc. 1998, 120 12216-12225; b) D. M. Rudkevich, G. Hilmersson, J. Rebek, J. Am. Chem. Soc. 1997, 119, 9911-9912.

[3] a) F. R. Pinacho Crisóstomo, A. Lledó, S. R. Shenoy, T. Iwasawa, J. Rebek, J. Am. Chem. Soc. 2009, 131, 7402-7410; b) R. J. Hooley, J. Rebek Jr, Chem. Biol. 2009, 16, 255-264.

[4] a) L. Catti, Q. Zhang, K. Tiefenbacher, Chem. Eur. J. 2016, 22, 9060-9066; b) M. Raynal, P. Ballester, A. Vidal-Ferran, P. W. N. M. van Leeuwen Chem. Soc. Rev. 2014, 43, 1734-1787; c) J. Meeuwissen, J. N. H. Reek, Nat. Chem. 2010, 2, 615-621.

[5] a) D. Vidal, M. Costas, A. Lledó, ACS Catal. 2018, 8, 3667-3672; b) A Lledo, A. Soler, Org. Chem. Front. 2017, 4, 1244-1249; c) A. Lledó, Org. Lett. 2015, 17, 3770-3773.

[6] a) S. Mosca, Y. Yu, J. V. Gavette, K.-D. Zhang, J. Rebek, J. Am. Chem. Soc 2015, 137, 14582-14585; b) S. M. Butterfield, J. Rebek Jr, Chem. Commun. 2007, 1605-1607; c) B. W. Purse, P. Ballester, J. Rebek, J. Am. Chem. Soc. 2003, 125, 14682-14683; d) J. Chen, J. Rebek, Org. Lett. 2002, 4, 327-329.

[7] S. Mecozzi, J. J. Rebek, Chem. Eur. J. 1998, 4, 1016-1022.

[8] a) L. Xu, G. Fang, J. Tao, Z. Ye, S. Xu, Z. Li, ACS Catal. 2018, 8, 11910 $11925 ;$ b) N.-W. Wu, I. D. Petsalakis, G. Theodorakopoulos, Y. Yu, J. Rebek, Angew. Chem. Int. Ed. 2018, 57, 15091-15095; Angew. Chem. 2018, 57, 15311-15315; c) C. Goehry, M. Besora, F. Maseras, Eur. J. Org. Chem. 2018, 2018, 2103-2109; d) H. Daver, A. G. Algarra, J. Rebek, J. N. Harvey, F. Himo, J. Am. Chem. Soc. 2018, 140, 12527-12537; e) H. Daver, J. N. Harvey, J. Rebek, F. Himo, J. Am. Chem. Soc. 2017, 139, 1549415503.

[9] a) L.-D. Syntrivanis, I. Némethová, D. Schmid, S. Levi, A. Prescimone, F. Bissegger, D. T. Major, K. Tiefenbacher, J. Am. Chem. Soc. 2020, 142, 5894-5900; b) E. Pahima, Q. Zhang, K. Tiefenbacher, D. T. Major, J. Am. Chem. Soc. 2019, 141, 6234-6246.

[10] a) A. Lledo, J. Rebek Jr, Chem. Commun. 2010, 46, 1637-1639; b) R. J. Hooley, S. R. Shenoy, J. Rebek, Org. Lett. 2008, 10, 5397-5400.

[11] K. Henzler-Wildman, D. Kern, Nature 2007, 450, 964.

[12] T. Gottschalk, P. D. Jarowski, F. Diederich, Tetrahedron 2008, 64, 83078317.

[13] D. F. Hahn, J. V. Milić, P. H. Hünenberger, Helv. Chim. Acta 2019, 102, e1900060.
[14] a) Y. I. Yang, Q. Shao, J. Zhang, L. Yang, Y. Q. Gao, J. Chem. Phys. 2019, 151, 070902; b) J. A. McCammon, Mol. Simul. 2016, 42, 1046-1055.

[15] L. Pesce, C. Perego, A. B. Grommet, R. Klajn, G. M. Pavan, J. Am. Chem. Soc. 2020, 142, 9792-9802.

[16] D. Hamelberg, J. Mongan, J. A. McCammon, J. Chem. Phys. 2004, 120, 11919-11929.

[17] a) C. García-Simón, C. Colomban, Y. A. Çetin, A. Gimeno, M. Pujals, E. Ubasart, C. Fuertes-Espinosa, K. Asad, N. Chronakis, M. Costas, J. Jiménez-Barbero, F. Feixas, X. Ribas, J. Am. Chem. Soc. 2020, 142, 1605116063; b) C. Curado-Carballada, F. Feixas, J. Iglesias-Fernandez, S. Osuna, Angew. Chem. Int. Ed. 2019, 58, 3097-3101; Angew. Chem. 2019, 58, 3129-3133; c) Y. Miao, F. Feixas, C. Eun, J. A. McCammon, J. Comput. Chem. 2015, 36, 1536-1549.

[18] J. R. Moran, J. L. Ericson, E. Dalcanale, J. A. Bryant, C. B. Knobler, D. J. Cram, J. Am. Chem. Soc. 1991, 113, 5707-5714.

[19] PM6-optimized starting geometries are available from the ioChem-BD repository: https://doi.org/10.19061/iochem-bd-4-26.

[20] a) A. Lledó, R. J. Hooley, J. Rebek, Org. Lett. 2008, 10, 3669-3671; b) P. Ballester, M. A. Sarmentero, Org. Lett. 2006, 8, 3477-3480.

[21] K. Nikitin, R. O'Gara, Chem. Eur. J. 2019, 25, 4551-4589.

[22] B. W. Purse, A. Gissot, J. Rebek, J. Am. Chem. Soc. 2005, 127, 1122211223.

[23] Gaussian 09, Revision B.01, M. J. Frisch, G. W. Trucks, H. B. Schlegel, G. E. Scuseria, M. A. Robb, J. R. Cheeseman, G. Scalmani, V. Barone, G. A. Petersson, H. Nakatsuji, X. Li, M. Caricato, A. Marenich, J. Bloino, B. G. Janesko, R. Gomperts, B. Mennucci, H. P. Hratchian, J. V. Ortiz, A. F. Izmaylov, J. L. Sonnenberg, D. Williams-Young, F. Ding, F. Lipparini, F. Egidi, J. Goings, B. Peng, A. Petrone, T. Henderson, D. Ranasinghe, V. G. Zakrzewski, J. Gao, N. Rega, G. Zheng, W. Liang, M. Hada, M. Ehara, K. Toyota, R. Fukuda, J. Hasegawa, M. Ishida, T. Nakajima, Y. Honda, O. Kitao, H. Nakai, T. Vreven, K. Throssell, J. A. Montgomery, Jr., J. E. Peralta, F. Ogliaro, M. Bearpark, J. J. Heyd, E. Brothers, K. N. Kudin, V. N. Staroverov, T. Keith, R. Kobayashi, J. Normand, K. Raghavachari, A. Rendell, J. C. Burant, S. S. lyengar, J. Tomasi, M. Cossi, J. M. Millam, M. Klene, C. Adamo, R. Cammi, J. W. Ochterski, R. L. Martin, K. Morokuma, O. Farkas, J. B. Foresman, and D. J. Fox, Gaussian, Inc., Wallingford CT, 2016.

[24] S. Miertuš, E. Scrocco, J. Tomasi, Chem. Phys. 1981, 55, 117-129.

[25] J. Wang, R. M. Wolf, J. W. Caldwell, P. A. Kollman, D. A. Case, J. Comput. Chem. 2004, 25, 1157-1174.

[26] A. Jakalian, B. L. Bush, D. B. Jack, C. I. Bayly, J. Comput. Chem. 2000, 21, 132-146.

[27] T. Darden, D. York, L. Pedersen, J. Chem. Phys. 1993, 98, 10089-10092.

[28] Y. Miao, W. Sinko, L. Pierce, D. Bucher, R. C. Walker, J. A. McCammon, J. Chem. Theory Comput. 2014, 10, 2677-2689.

Manuscript received: February 12, 2021

Accepted manuscript online: April 21, 2021

Version of record online: 


\section{FULL PAPER}

The complex dynamic behavior of a self-folding cavitand receptor has been thoroughly characterized employing molecular dynamics simulations and ${ }^{1} \mathrm{H}$ EXSY experiments. The exchange barriers extracted from accelerated molecular dynamics simulations are in very good agreement with experimentally determined values. Overall, the presented approach emerges as a useful tool for the rational design of dynamic host-guest systems.

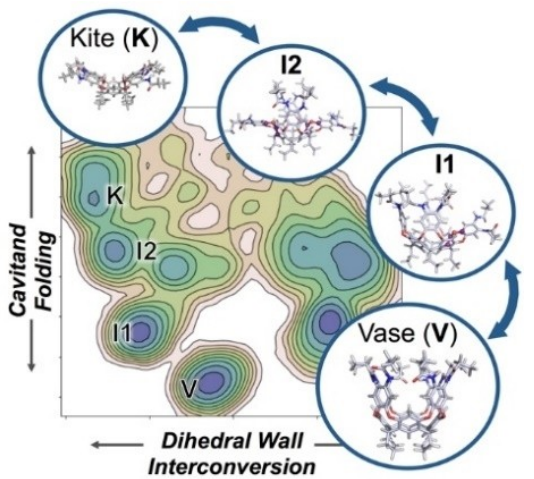

R. López-Coll, R. Álvarez-Yebra, Dr. F. Feixas*, Dr. A. Lledó*

$1-9$

Comprehensive Characterization of the Self-Folding Cavitand Dynamics 